\title{
Low Temperature Growth of Single-Walled Carbon Nanotubes by Water Plasma Chemical Vapor Deposition
}

\author{
Yo-Sep Min, Eun Ju Bae, Byung Seok Oh, Donghun Kang, and Wanjun Park* \\ Materials \& Devices Research Center, Samsung Advanced Institute of Technology, \\ Yong-In, Kyeongki-Do, 449-712, Korea \\ E-mail:wanjun@samsung.com
}

\section{Supporting Information}

\section{Growth of SWNTs}

A homemade radio frequency $(13.56 \mathrm{MHz})$ remote PECVD system was used for nanotube growth. A quartz tube with a 2-inch diameter was surrounded by a copper coil in a helical manner from the gas inlet to the sample holder which was located at a distance of $17 \mathrm{~cm}$ from the end of the copper coil. Deionized water was charged to a canister attached to the quartz tube. Water was evaporated and carried to the quartz tube by the vacuum without any carrier gas. The amount of water vapor was regulated by a metering valve and monitored with pressure increase in the quartz tube. Substrates were heated by halogen lamp and the growth temperature was calibrated with a thermocouple-implanted quartz holder. The calibrated temperature was not influenced by the remote plasma ignition.

Iron catalyst-deposited substrates $\left(\mathrm{SiO}_{2} 400 \mathrm{~nm} / \mathrm{Si}\right)$ were placed on a quartz holder and then heated up to the growth temperature for $300 \mathrm{sec}$ in water atmosphere which results in an increase of 5 mTorr in the base pressure. After an interval for the stabilization of substrate temperature, methane $(60 \mathrm{sccm})$ was introduced into the quartz tube and the subsequent plasma was ignited to grow CNTs. During the CNT growth $(20 \sim 600 \mathrm{sec})$, the working pressure was maintained at $\sim 0.37$ Torr. CNT growth was performed at $450{ }^{\circ} \mathrm{C}$ with a plasma power of $15 \mathrm{~W}$.

\section{Fabrication of Network FETs}

Source and drain electrodes were fabricated on the SWNT-grown substrate (density $\sim 30 / \mu \mathrm{m}^{2}$ ) using optical lithography and lift-off of $\mathrm{Ti}(10 \mathrm{~nm}) / \mathrm{Au}(100 \mathrm{~nm})$ films. The specimen was then dipped in buffered oxide etch (6:1) for $90 \mathrm{sec}$ to remove nanotubes outside the regions of the devices between the source-drain electrodes. The fabricated network transistors have a channel length of $5 \mu \mathrm{m}$ and a width 
of $40 \mu \mathrm{m}$. The density of nanotubes was evaluated to be $\sim 30 / \mu \mathrm{m}^{2}$ from scanning electron microscopic (SEM) image as shown in Figure S1a.

\section{Comparison of Densities of Nanotubes Grown by Water Plasma and Argon Plasma CVD}

Figure S1 shows SEM images of nanotubes grown by water plasma (a) and argon plasma (b) CVD. For the specimens in the SEM images, all process conditions for catalyst preparation and nanotube growth are same except that for Fig. S1a, water was introduced to the quartz tube to ignite plasma instead of argon used for Fig. S1b. Catalytic nanoparticles were deposited on $\mathrm{SiO}_{2} / \mathrm{Si}$ substrates from 0.05 M solution of ferrocene and AZ5214E (Ref. 12). Nanotube growth was performed at $450{ }^{\circ} \mathrm{C}$ with a plasma power of $15 \mathrm{~W}$ for $3 \mathrm{~min}$. These images clearly reveal that the density of nanotubes was dramatically increased by using water plasma.
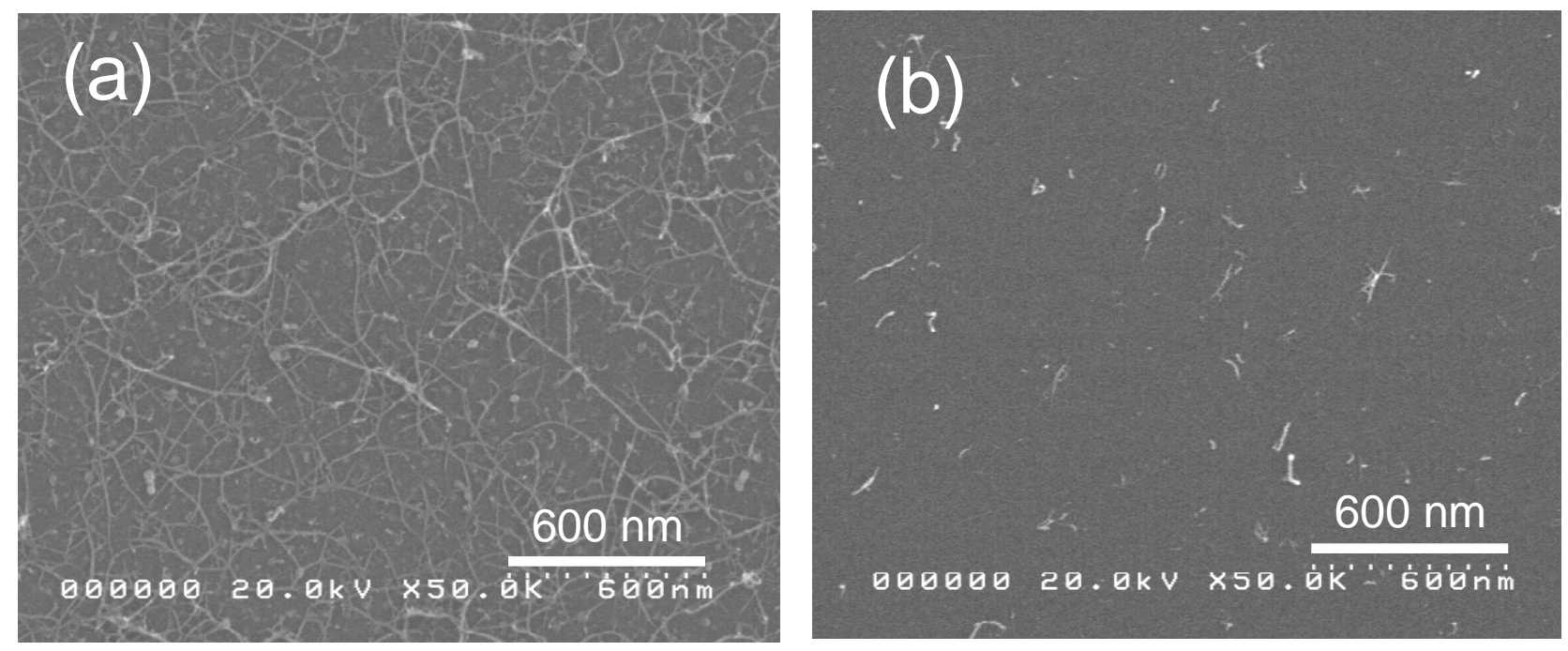

Figure S1. SEM images of SWNTs grown by water plasma (a) and argon plasma (b) CVD.

The density increase of SWNTs is proven with Raman spectra as shown in Figure S2. The intensities of G-band and RBM peaks were also dramatically increased due to the density increase of SWNTs by water plasma. In the case of argon plasma, the G-band peak is much weaker than the substrate peak at $521 \mathrm{~cm}^{-1}$, and the intensity of RBM peak is comparable to that of the substrate peak at $303 \mathrm{~cm}^{-1}$. However, the specimen grown by water plasma shows strong G-band of which the intensity is comparable to the substrate peak at $521 \mathrm{~cm}^{-1}$, and the RBM peak is much stronger than the peak at 303 $\mathrm{cm}^{-1}$. 


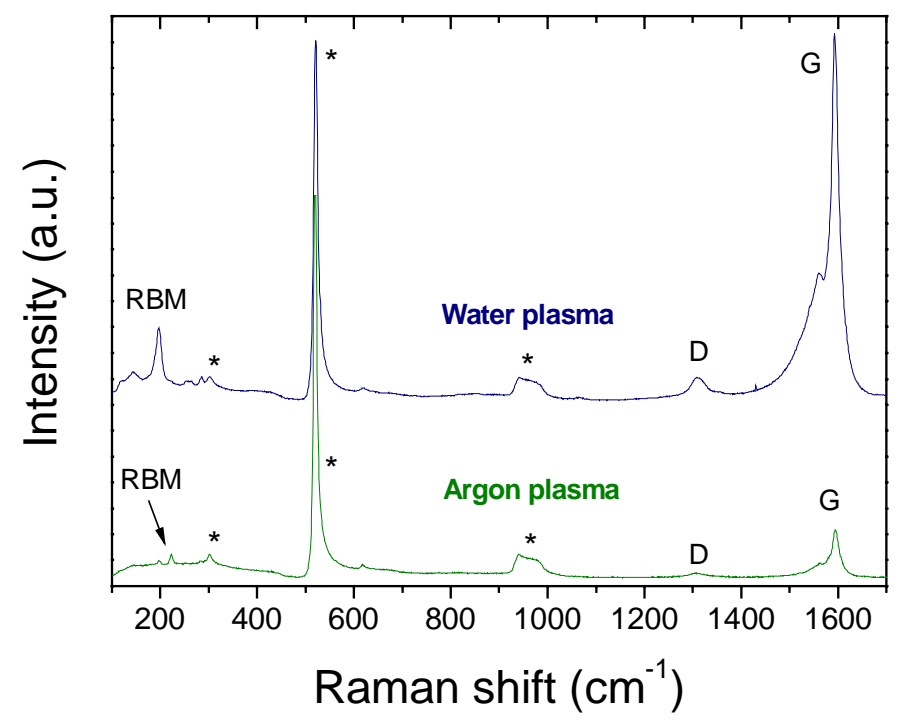

Figure S2 shows the Raman spectra $(633 \mathrm{~nm})$ of the specimens shown in Fig. S1. 